\title{
Research on Computer Network Security and Its Countermeasures
}

\author{
Li Jian \\ Yunnan Jiaotong College, Kunming, 650500
}

Keywords: computer; network security; countermeasure research

\begin{abstract}
With the continuous development of economy and science, computer network has become an indispensable part of people's life and work. The big data age requires us to master computer skills and make use of large data to help us more easily and comfortably, but everything has its double side. After the computer network technology has helped us get more comfortable and convenient working environment, the network security problems also follow, some people start to use the loopholes in the network to steal information, causing network security problems, and people's work and life have also brought great worries, if personal data is out of the outside. Therefore, there is no guarantee for personal safety and property safety. How to solve the problem of computer network security at this stage is what we are most concerned about.
\end{abstract}

\section{Introduction}

with the continuous development of science and technology, in order to effectively improve the quality of work and work efficiency, and maximize the maximum amount of things in a limited period of time, many enterprises have chosen computer computing to manage in the process of daily work, but if they are in the process of use, it will be planned. The security of the computer is not guaranteed effectively, so once the information theft is done, it will cause great losses. Therefore, how to protect computer network security has always been a problem we need to think about and solve. Nowadays, there are many enterprises in the market competition in order to seize the opportunity to seize the opportunity to use the Internet to steal information from other enterprises, such things are common. Some people even make malicious computer viruses to steal other people's property and information, so strengthening network security is one of the things we must do at present.

\section{The Safety Problems of Computer Network}

The so-called computer network security problem, the main point is that at the present stage someone uses the loopholes in the network system to unlawfully invade to steal other people's information in exchange for profit. The exposure of personal information and enterprise information to a certain extent has led to the instability of the social and market economy. As time goes on, the problem of computer network security is not only not subsided, but it has more and more intense signs. In order to ensure the user's information security, it is very necessary to find the existing problems. At present, there are several problems in the current computer network security[1].

The main point of the so-called physical problem is that the computer network system has a recognition error in the process of identifying the identity, such as the identification of the theft or the espionage. Nowadays, there are many illegal elements to use the network to steal information, especially with the continuous development of the market economy and the increasing pressure of competition in the market today, more and more enterprises will be stolen by illegal elements.

In the present stage, the most serious of computer network security technology is the continuous emergence of loopholes in the network system, some web designers have ignored the problem of network security in the process of programming, which directly lets the unlawful elements to be used. In order to effectively prevent this problem, users can update and repair the network system regularly.

At present, with people's attention to network security, more and more people in the process of 
computer use will set private user name and password to ensure the security of the computer, but in the process of using the network, there are many people who will be troublesome in the process of setting up password and password. A single password, such as direct use of your own birthday, can cause an intruder to easily crack your password, so the choice of a complex and difficult password can effectively protect your personal information[2].

At present, the problem of cable connection often happens, which mainly includes different contents such as eavesdropping, pseudo substitution and so on. Especially when people are using computers, eavesdropping happens frequently, and the frequency at radio stations and TV stations is even higher.

Nowadays, many people install virus in software. Once the software is installed on the computer, the virus will follow, so it is very necessary to download software on the web page, or to test it after downloading the software, so as to avoid the virus invasion caused by the software.

\section{Solutions to the Problems of Computer Network Security}

Unlike other science and technology, computer network technology has its own great openness and inclusiveness, which is its advantage and its biggest disadvantage, because the network system is very vulnerable to security vulnerabilities. Once the computer network system has loopholes, the security and information within the system are destroyed. In the process of using, people are likely to meet the problem of incomplete information or information being tampered with, and it is easy to cause the waste of resources. To solve this problem, the computer appears at the present stage. The problem of network security is very important.

The security of the computer network system is mainly created and maintained by the development of computer network and the writers, which directly requires the staff to maintain a high concentration of attention during the process of work and establish a correct awareness of protection. The security of the system is in the first place. After the completion of the program, it needs to test it repeatedly, to find out the network security problems that are ignored in the process of writing, and to repair and solve the problems in order to reduce the security risks existing in the system. In the present environment, the speed of network development is very fast, new viruses can appear every day. The speed of the virus transmission is very fast, and the damage to the computer is very large. In the process of using the computer network, the user needs to use good killing software and check the computer regularly. To avoid the existence of a virus is not known. At the same time, for the manufacturer of antivirus software, we should know the current network environment in real time and update the antivirus software regularly to ensure the efficiency of the antivirus software and the ability to protect the computer. Users need to backup important data before the computer network system runs, so as not to cause unnecessary economic loss caused by the loss of data caused by the virus. At the same time, it needs to update the antivirus software regularly. After the download of other software is completed, it is necessary to use antivirus software to confirm it, so as to avoid it. The virus is carried in the medium. In the process of applying the computer network, do not random point open unknown files or random points open the interface of the network section suddenly pop-up, update the computer protection system and patch in time to protect the network security from all aspects, help us not to be safe in the process of work and life. All the trouble[3].

To better guarantee the security of the computer network we use, in addition to the need for network development and writing staff to improve security awareness in the process of developing and writing, it is also necessary to create a secure, stable network use network management staff who can use the router to do so The network system is supervised, managed and controlled in real time. In the process of using the user, the user needs to be authenticated. It requires each user to enter and use the system only through authentication, and the failure of identity authentication can not be used. Once the identity authentication fails more than 3 times, it needs to be used. Households lock for 30 minutes in order to reduce the registration of lawless elements. At the same time, users who have already passed authentication need to restrict their rights, so they can not do whatever they want. More important is to strengthen the user's own network security awareness, the 
use of the network before the use of appropriate knowledge of the network security, as far as possible to avoid network fraud and other problems, once the network fraud personnel can immediately be reported to the related network police to report. What the user needs to do in the process of using the network system is to do the correct operation according to the rules and regulations, and avoid the effective regulation of the user's network operation. Only in this way can we ensure the security and stability of the network environment in the process of using the Internet as far as possible.

With the development of economy and science and technology, the application of computer network technology in our daily work is very common. Our life and work are gradually inseparable from the use of the network. A lot of information and data are directly placed in the computer, and many hackers try to use the computer network. To steal information from people's computers and sell them to make profits. Therefore, in order to better protect the data information in the computer, we must encrypt the data information in the computer, especially for some confidential information inside the computer, we need to use the highest level of technology to protect it. Nowadays, because of the competitiveness of the market, many enterprises in order to better develop their own enterprises to press other enterprises, using the loopholes in the computer network to steal and destroy other people's data information, this kind of thing is common, which is not good for the market and economic development, if the enterprise only wants to use it. In this way to win the market economy, rather than the real improvement of the business level and innovation ability of their own enterprises, the overall economy of the society will continue to decline. In order to avoid this problem effectively, it is necessary to encrypt the data information in the computer, and it is necessary to set the public key or set the private key to the computer according to its own actual situation, in order to protect the data inside the computer from being destroyed. Only by increasing the protection of computer data, can the data we need and use are always in a safe and comfortable environment [4].

From the present environment and technology, it is very important to apply the firewall technology in the computer network technology, and the firewall technology is one of the most effective measures to ensure the network security technology of the computer at the present stage. Firewall technology is widely used in the process of use at the present stage. Using firewall technology can not only effectively monitor the user's data use in different periods of time, but also can accurately record the main operating information and creation state of the user in the process of use, and use fireproof paint. The technology can establish an effective and powerful monitoring system within the computer, and can effectively monitor the security and state of the computer network, and can find out the problem at any time to solve the problems that have already appeared. Therefore, in the process of using the computer network, we can use the firewall technology to supervise the internal system of the computer. Before the use of the computer, we need the user to $\log$ in. In the process of logging in, its own system will strictly audit all the information of the user to ensure the safety of the use. In the process of login, only the user's input password and account can be used when the user is set up in the system before. In the process of use, the user can only perform the system operation to a certain extent. At present, the use of this technology can improve the problem of network security management. The security of computer network also ensures the information security of users, and further improves the reliability of communication security [5].

The so-called network digital certificate and signature technology, which mainly refers to the use of third parties to the users of the network system at the present stage to verify the user identity, to ensure that any user's personal information will not be leaked to the user to verify the identity of the user. This method can reduce the appearance of illegal elements more effectively, and reduce the occurrence of illegal elements using the loopholes in the network system to log in in order to steal information. At the same time, using this technology can help us better protect the problem of network information and the storage of computer information. In the process of information retrieval, we can use the network signature technology to let the user first confirm the signature and send the inspection when reading the information, if it is different from the original information three times. The above can be used to lock the user's account in order to ensure the security of the 
user's information, so that the security of the network environment can be maintained to ensure that we have a secure, comfortable network environment, better use of the network to work and learn. However, the current technology needs to be developed and there are many computers at this stage. Without the function of opening the network signature, the technology can not be carried out without a handwritten keyboard. The application of this technology is closely related to the development of computer technology. In order to better protect the security of the computer network, we need to develop technology and innovate technology as much as possible [6].

\section{Conclusion}

According to this article, we can know that in the present environment, network security has become one of the most important things in our daily life. At the same time, it is also one of the concerns of all walks of life. However, at present, there are still some questions in the security of computer network in China. How to solve these problems is the most important. Only by ensuring the security of the computer network can we ensure the security of the people's information. At the same time, it can help the enterprises to develop better, prevent the network crime and other problems. Therefore, it is very important to improve the network security. Not only need the user's own main information not to be exposed at random. As a network manager, it is more necessary to improve the level of network security and protection technology, give users a safe network environment, let people use the network to work and study safely, and make better use of their own material for the economic development of our country. Contribution.

\section{References}

[1] Liu H. Research on Computer Network Security and Its Countermeasures[J]. China Computer \& Communication, 2017.

[2] Zou $\mathrm{Y}, \mathrm{Bu} \mathrm{R}$, Zou $\mathrm{Y}$, et al. Computer network information security and Its Countermeasures[C]// International Conference on Mechatronics, Materials, Chemistry and Computer Engineering. 2017.

[3] Zhao L M. Research on network security risk analysis and countermeasures[J]. Journal of Hunan City University, 2016.

[4] Kumar G S, Sirisha C V K, Kanaka D R, et al. Web Users Session Analysis Using DBSCAN and Two Phase Utility Mining Algorithms[J]. International Journal of Soft Computing \& Engineering, 2012, 1(6).

[5] Liang K. Exploration of the Security Risks Facing Computer Networks and Its Countermeasures[J]. Journal of Jiangsu Vocational \& Technical Institute of Economics \& Commerce, 2012.

[6] Zhang N, Zhu K, Zhu D. Research on DoS attack and its prevention countermeasures[J]. China Internet, 2016.

A brief introduction to the author: Li Jian (1976.7), male, Hani, Yunnan Kunming, master, Yunnan Jiaotong College, associate professor, research direction: computer network technology, traffic intelligent control 